\title{
Aspirin Related Platelet Reactivity as a Determinant of Ten Year Survival in High Risk Non-ST segment Elevation Myocardial Infarction (NSTEMI) Patients
}

Authors: Nazish Khan ${ }^{1}$, Mamas A Mamas², Alexandra Moss ${ }^{1}$, Diana A Gorog ${ }^{3}$, Peter Nightingale $^{4}$, Angel Armesilla ${ }^{5}$, Andrew Smallwood ${ }^{1}$, Shahzad Munir ${ }^{1}$, Saib Khogali ${ }^{1}$, Ben Wrigley ${ }^{1}$, James M Cotton ${ }^{1,5}$

1. Department of Cardiology, Heart and Lung Centre, New Cross Hospital, Wolverhampton, West Midlands, WV10 0QP, England.

2. Keele Cardiovascular Research Group, Keele University, Stoke-on-Trent, United Kingdom; Department of Cardiology, Royal Stoke University Hospital, Stoke-on-Trent, United Kingdom.

3. University of Hertfordshire, Hertfordshire, United Kingdom; Imperial College, London, United Kingdom.

4. Institute of Translational Medicine, Heritage Building (Queen Elizabeth Hospital) University Hospitals Birmingham NHS Foundation Trust, Birmingham, B15 2TH.

5. Research institute in Healthcare Science, Faculty of Science and Engineering, University of Wolverhampton.

Address for Correspondence: Dr Nazish Khan, Department of Cardiology, Heart and Lung Centre, The Royal Wolverhampton Hospitals NHS Trust, Wednesfield Road, Wolverhampton, WV10 0QP

Tel: $0044(0) 1902694218$

Fax: $0044(0) 1902695646$

Email: nazish.khan@nhs.net

Research Letter to the Editor, word count: 1,354

Key Words: NSTEMI, Aspirin resistance, mortality 
Letter to the Editors:

Aspirin, the most commonly prescribed antiplatelet agent in clinical practice, forms a cornerstone of management in patients with established cardiovascular disease (CVD). The clinical efficacy and safety of aspirin when prescribed for secondary prevention is supported by a robust evidence base demonstrating a $24 \%$ reduction in mortality and a $25 \%$ reduction in serious adverse events without any increase in bleeding complications in the context of an acute myocardial infarction [1]. Nevertheless, numerous studies have shown that the antiplatelet effect of aspirin is not uniform and is often sub-optimal in a sizable proportion of patients [2, 3]. Inadequate platelet inhibition following the administration of aspirin is associated with recurrent cardiovascular events and adverse outcomes in patients with coronary artery disease [2].

Dual antiplatelet therapy (DAPT) in the form of aspirin and a P2Y12 inhibitor has proven benefit for the prevention of atherothrombotic complications in both stable and high-risk patients [4]. However, despite the initiation of DAPT, up to $20 \%$ of patients experience a recurrent ischaemic event within a year of their index presentation and in non-ST-elevation myocardial infarction (NSTEMI) mortality at five years is $22 \%$ [5].

This ongoing residual ischemic risk has led to significant interest in the area of antiplatelet treatment failure. Much of this focus has recently been aimed at overcoming the perceived limitations associated with the P2Y12 inhibitor clopidogrel (an agent with significant pharmacokinetic and pharmacodynamic variability in-vivo). The development and adoption of more potent P2Y12 inhibitors (i.e. ticagrelor and prasugrel) which provide faster, greater and more consistent levels of platelet 
inhibition has led to lower recurrent ischaemic events and cardiovascular mortality [6, 7]. Irrespective of the P2Y12 inhibitor under investigation, the pivotal landmark DAPT clinical trials were all undertaken with aspirin as the background anti-platelet agent of choice. Whilst the default duration of treatment for DAPT is 12 months, the long term clinical utility and implications of a sub-optimal response to aspirin monotherapy following discontinuation of the P2Y12 inhibitor after this 12-month period have been largely under explored.

In the absence of a universally accepted definition, the concept of aspirin resistance is difficult to define and reported prevalence ranges from $5.5 \%$ to $61 \%$ depending on the patient group studied and the technique used to measure aspirin activity [3]. This reduced aspirin responsiveness has been shown to be associated to adverse clinical events in the short to medium term [2]. In clinical practice, quantitative assessment of the degree of aspirin induced platelet inhibition is readily available using the VerifyNow system (Accriva, San Diego, Ca). Using this device aspirin resistance units $(A R U)$ of $\geq 550$ indicates a poor response and thereby high on treatment platelet reactivity (HPR) [8]. Little is known about the importance of relative aspirin resistance over a long time-course. We sought to investigate whether the impact of VerifyNow determined response to aspirin, even at levels that were previously thought to indicate an adequate antiplatelet response, is prognostically important in terms of all-cause mortality at 10 years.

We performed a single centre, prospective, observational study of 224 patients admitted with NSTEMI undergoing coronary angiography. Patients were followed up for a period of 120 months (10 years) for the primary end point of survival at 10 years, 
expressed as all-cause mortality. The study was approved by the local research ethics committee and conducted in accordance with the principles of the Declaration of Helsinki. All patients gave written informed consent prior to inclusion. Mortality data were obtained from the United Kingdom Office of National Statistics to determine the patients' vital status at 10 years following the index admission.

Aspirin-naïve patients were administered an oral loading dose of aspirin $300 \mathrm{mg}$ and maintained on aspirin $75 \mathrm{mg}$ daily thereafter. In line with contemporary recommendations at that time, all patients were administered an oral loading dose of clopidogrel $300 \mathrm{mg}$ daily and maintained on clopidogrel $75 \mathrm{mg}$ daily for 12 months only. The degree of inhibition of platelet reactivity following the administration of aspirin was assessed using the point of care VerifyNow Aspirin assay and expressed as ARU (Accriva, San Diego, Ca).

The mean time between administration of an aspirin loading dose (or aspirin on admission) to angiography was $4.9 \pm 2.7$ days, reflecting practice in $2007 / 8$. Of this group 54\% ( $\mathrm{n}=122)$ underwent $\mathrm{PCl}$ and stent implantation. Baseline clinical characteristics and cardiovascular risk factors, in relation to tertiles of aspirin-effect (expressed as ARU) are presented in Table 1. All three groups were well matched for baseline characteristics and cardiovascular risk factors. Patients in T1 (ARU 363 -405) tended to be younger than those in T2 (ARU $406-436$ ) and T3 (ARU 437 -596). Although not of statistical significance, a difference was also noted in the number of diabetic patients within each tertile, with a greater proportion in T2 and T3. 
The effect of high on aspirin platelet reactivity on long term survival is shown in figure 1, with higher ARU values associated with increased mortality (log rank, $p=0.009)$. Whilst diabetes is known to adversely impact on platelet reactivity following the administration of aspirin, a sub-group analysis of our non-diabetic cohort indicates that the difference in all-cause mortality observed is not driven by diabetic status alone, since a statistically significant difference was also observed in non-diabetic patients (log rank, $p=0.005)$. A cox regression analysis demonstrated that age at presentation $(\mathrm{HR}=1.098,95 \% \mathrm{Cl}[1.05-1.14], \mathrm{p}<0.0001)$ in addition to HTPR (according to the current definition of $A R U \geq 550)$ at angiography $(H R=3.03,95 \% \mathrm{Cl}[1.38-6.99], p=$ 0.009), were statistically significant predictors of mortality at 10 years. Both coxregression and ROC analyses (c-statistic > 0.70) demonstrate that ARU values as low as 406 are associated with reduced long-term survival.

Our study is the first to determine the clinical significance of aspirin related platelet reactivity at the time of angiography on longer-term survival in patients presenting with NSTEMI. We demonstrate that ARU values lower than the previously defined cut off $(A R U \geq 550)$ are associated with reduced survival when assessed over this extended follow up period. Our findings challenge the current clinical paradigm that aspirin resistance is a dichotomous variable which might lead patients to be at increased short-term risk of recurrent cardiovascular events. In our cohort of high risk NSTEMI patients investigated with in-patient coronary angiography, our results suggest a continuum of risk related to aspirin related platelet activity. Patients in the second and third tertile (i.e. ARU above 406) had significantly increased mortality at 10 years, compared to those in tertile 1 (log rank, $p=0.009$ ). 
Recent publications have highlighted the benefit of extended duration or additional antithrombotic therapies in patients with established cardiovascular disease at high ischaemic risk $[9,10]$. These studies lend support to the concept that aspirin monotherapy is insufficient to prevent atherothrombotic events in a significant number of patients with chronic coronary syndrome. Aspirin responsiveness may be a simple way of identifying such high-risk individuals who may benefit from additional pharmacotherapies, whilst avoiding bleeding risk due to the use enhanced antithrombotic medication in low risk patients.

Several limitations of the present study should be mentioned. Our analysis represents a single centre experience of the assessment of platelet reactivity in a relatively small number of aspirin treated patients. Platelet reactivity following the administration of aspirin is assessed using only a single platelet function assay (VerifyNow) at a single time point (the time of in-patient angiography) and may be due to heightened platelet reactivity secondary to an ACS event. We have limited data regarding on-going adherence to prescribed secondary prevention therapy, and have assumed that all patients have continued to receive and be adherent to secondary prevention medications, including aspirin. Our follow up results at 10 years are limited to vital status only and we do not have information regarding recurrent ischaemic events or bleeding complications.

In conclusion, the anti-platelet effect of aspirin, when measured early in patients with high risk NSTEMI, predicts survival at 10 years. Levels of platelet response previously thought to be adequate indicate significant ongoing risk of mortality. This simple measurement of aspirin responsiveness at the time of index presentation might 
identify a sub-group of patients in which further optimisation and intensification of their antithrombotic treatment regime may be warranted.

Table 1: Baseline characteristics, cardiovascular risk factors, patient therapy, procedural characteristics and medications on discharge for troponin positive NSTEMI patients expressed according to tertiles of platelet reactivity.

\begin{tabular}{|c|c|c|c|c|}
\hline & T1 $(n=76)$ & T2 $(n=76)$ & T3 $(n=72)$ & p-value \\
\hline Age & $\begin{array}{l}61.12 \pm \\
11.00\end{array}$ & $\begin{array}{l}64.46 \pm \\
10.47\end{array}$ & $\begin{array}{l}64.90 \pm \\
10.40\end{array}$ & 0.06 \\
\hline Sex (male) & $52(68)$ & $48(63)$ & $52(72)$ & 0.494 \\
\hline \multicolumn{5}{|l|}{ Risk Factors } \\
\hline Diabetes & $9(12)$ & $16(21)$ & $20(28)$ & 0.057 \\
\hline Hypertension & $36(47)$ & $46(61)$ & $43(60)$ & 0.153 \\
\hline Current Smoker & $20(26)$ & $22(29)$ & $22(31)$ & 0.603 \\
\hline Previous Ml & $15(20)$ & $22(29)$ & $21(29)$ & 0.346 \\
\hline Previous CABG & $3(4)$ & $5(7)$ & $4(6)$ & 0.779 \\
\hline Previous TIA/Stroke & $3(4)$ & $7(9)$ & $5(7)$ & 0.440 \\
\hline Positive FHx & $32(42)$ & $37(49)$ & $32(44)$ & 0.717 \\
\hline Hypercholesterolaemia & $33(43)$ & $45(59)$ & $37(51)$ & 0.125 \\
\hline \multicolumn{5}{|l|}{ Patient Therapy } \\
\hline $\mathrm{PCl}$ & $46(61)$ & $44(58)$ & $33(46)$ & 0.162 \\
\hline Medical management & $17(23)$ & $21(28)$ & $21(28)$ & 0.613 \\
\hline CABG & $8(11)$ & $11(14)$ & $11(14)$ & 0.139 \\
\hline $\begin{array}{l}\text { Normal coronary } \\
\text { arteries }\end{array}$ & $5(6.5)$ & $0(0)$ & $2(3)$ & 0.065 \\
\hline \multicolumn{5}{|l|}{$\mathrm{PCl}$ characteristics } \\
\hline Single vessel & $39(51)$ & $46(61)$ & $20(26)$ & 0.010 \\
\hline Two vessel & $5(7)$ & $3(4)$ & $8(11)$ & 0.022 \\
\hline Triple vessel & $1(1)$ & $0(0)$ & $1(1)$ & 0.470 \\
\hline BMS & $15(20)$ & $15(20)$ & $12(16)$ & 0.619 \\
\hline DES & $32(42)$ & $34(45)$ & $18(24)$ & 0.701 \\
\hline \multicolumn{5}{|c|}{ Medication on discharge } \\
\hline Beta-blocker & $54(71)$ & $58(76)$ & $53(74)$ & 0.675 \\
\hline ACE inhibitor & $60(79)$ & $53(70)$ & $50(70)$ & 0.405 \\
\hline Statin & $72(95)$ & $72(95)$ & $70(97)$ & 0.445 \\
\hline Aspirin & $73(96)$ & $73(96)$ & $70(97)$ & 0.883 \\
\hline Clopidogrel & $63(83)$ & $72(95)$ & $63(91)$ & 0.034 \\
\hline
\end{tabular}

Values are expressed as mean \pm standard deviation, frequency $(\%)$. $\mathrm{Ml}=$ myocardial infarction, $\mathrm{CABG}=$ coronary artery bypass graft, $\mathrm{TIA}=$ transient ischaemic attack, $\mathrm{FHx}=$ family history of premature cardiovascular disease, $\mathrm{PCl}=$ percutaneous coronary intervention, $\mathrm{BMS}$ = bare metal stent, $\mathrm{DES}=$ drug eluting stent, $\mathrm{ACE}=$ angiotensin converting enzyme 
Figure 1: Kaplan-Meier curve demonstrating the impact of the degree of inhibition of platelet reactivity following the administration of aspirin (expressed as ARU) as a predictor of long-term survival in troponin positive NSTEMI patients.

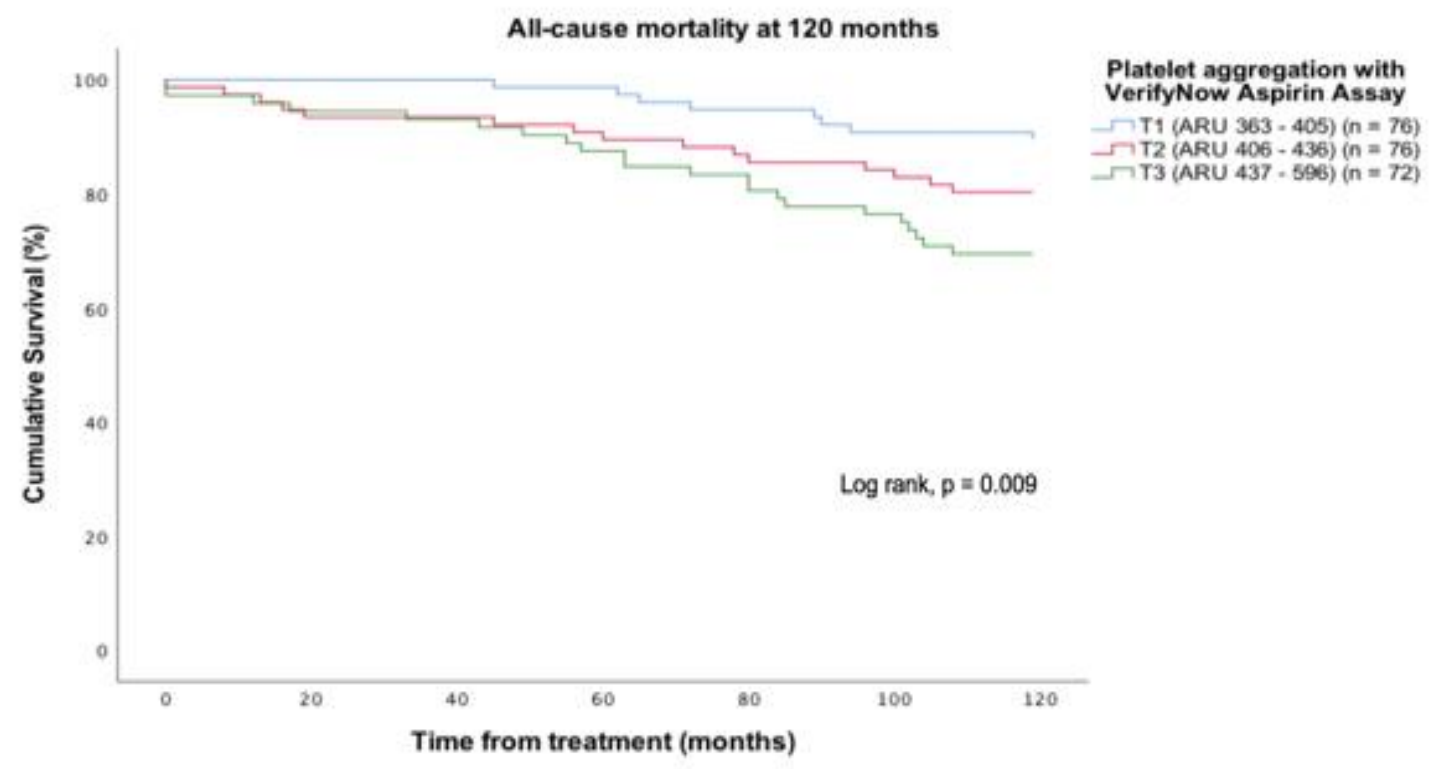

References: 
1. ISIS-2CollaborativeGroup, Randomized trial of intravenous streptokinase, oral aspirin, both, or neither among 17,187 cases of suspected acute myocardial infarction: ISIS-2.ISIS-2 (Second International Study of Infarct Survival) Collaborative Group. J Am Coll Cardiol, 1988. 12(6 Suppl A): p. 3A-13A.

2. Chen, W.H., et al., Aspirin resistance and adverse clinical events in patients with coronary artery disease. Am J Med, 2007. 120(7): p. 631-5.

3. Lordkipanidze, M., et al., A comparison of six major platelet function tests to determine the prevalence of aspirin resistance in patients with stable coronary artery disease. Eur Heart J, 2007. 28(14): p. 1702-8.

4. Bhatt, D.L., et al., Patients with prior myocardial infarction, stroke, or symptomatic peripheral arterial disease in the CHARISMA trial. J Am Coll Cardiol, 2007. 49(19): p. 1982-8.

5. Fox, K.A., et al., Underestimated and under-recognized: the late consequences of acute coronary syndrome (GRACE UK-Belgian Study). Eur Heart J, 2010. 31(22): p. 2755-64.

6. Wallentin, L., et al., Ticagrelor versus clopidogrel in patients with acute coronary syndromes. N Engl J Med, 2009. 361(11): p. 1045-57.

7. Wiviott, S.D., et al., Prasugrel compared with high loading-and maintenance-dose clopidogrel in patients with planned percutaneous coronary intervention: the Prasugrel in Comparison to Clopidogrel for Inhibition of Platelet Activation and Aggregation-Thrombolysis in Myocardial Infarction 44 trial. Circulation, 2007. 116(25): p. 2923-32.

8. Nielsen, H.L., et al., Aspirin response evaluated by the VerifyNow Aspirin System and light transmission aggregometry. Thromb Res, 2008. 123(2): p. 267-73.

9. Eikelboom, J.W., et al., Rivaroxaban with or without Aspirin in Stable Cardiovascular Disease. N Engl J Med, 2017. 377(14): p. 1319-1330.

10. Bonaca, M.P., et al., Long-term use of ticagrelor in patients with prior myocardial infarction. N Engl J Med, 2015. 372(19): p. 1791-800. 